\title{
Novel read density distribution score shows possible aligner artefacts, when mapping a single chromosome
}

\author{
Fedor M. Naumenko ${ }^{1 *}$, Irina I. Abnizova ${ }^{2,3}$, Nathan Beka ${ }^{4}$, Mikhail A. Genaev ${ }^{5}$ and Yuriy L. Orlov ${ }^{1,5,6^{*}}$ \\ From Belyaev Conference \\ Novosibirsk, Russia. 07-10 August 2017
}

\begin{abstract}
Background: The use of artificial data to evaluate the performance of aligners and peak callers not only improves its accuracy and reliability, but also makes it possible to reduce the computational time. One of the natural ways to achieve such time reduction is by mapping a single chromosome.

Results: We investigated whether a single chromosome mapping causes any artefacts in the alignments' performances. In this paper, we compared the accuracy of the performance of seven aligners on well-controlled simulated benchmark data which was sampled from a single chromosome and also from a whole genome. We found that commonly used statistical methods are insufficient to evaluate an aligner performance, and applied a novel measure of a read density distribution similarity, which allowed to reveal artefacts in aligners' performances. We also calculated some interesting mismatch statistics, and constructed mismatch frequency distributions along the read.

Conclusions: The generation of artificial data by mapping of reads generated from a single chromosome to a reference chromosome is justified from the point of view of reducing the benchmarking time. The proposed quality assessment method allows to identify the inherent shortcoming of aligners that are not detected by conventional statistical methods, and can affect the quality of alignment of real data.
\end{abstract}

Keywords: Next-generation sequencing, DNA alignment, Read density distribution

\section{Background}

In the last few years next-generation sequencing (NGS), also known as high-throughput sequencing, has shown an impressive amount of development due to significantly increased throughput accompanied by plunging costs. The production of gigabytes of sequencing data in a few hours presents ever-increasing demands on the quality and speed of processing [1].

Mapping reads against a reference genome is typically the first step in analyzing next-generation sequencing data. It is an important step for further analysis, such as identification of protein binding sites from ChIPsequencing data or variant calling. The quality and

\footnotetext{
* Correspondence: fedor.naumenko@gmail.com; orlov@bionet.nsc.ru ${ }^{1}$ Novosibirsk State University, Pirogova, 1, Novosibirsk 630090, Russia Full list of author information is available at the end of the article
}

efficiency of mapping becomes critical. Hence the growing interest in benchmarking of short read aligners is clear.

A number of robust benchmarking surveys of short read aligners have been published [2-9]. These surveys focus on evaluating of such aspects of mapping accuracy as number of correctly and incorrectly mapped reads, percentage of multi-mapped hits (it will be explained later in the text), variation and errors (single nucleotide polymorphism, splicing, inserts and deletions rate etc.), and technical features (execution time and randomaccess memory). To reliably estimate these tools, simulated data is often used because of their predictability, reproducibility and manageability.

In particular, manageability is manifested in the fact that artificial sequence can be constructed on the basis of a single chromosome. It is natural to assume that the 
aligning of such data requires time that is reduced as minimum in proportion to the ratio of the chromosome length to the whole genome length. Moreover, further processing (peak calling, etc.) is performed independently for each chromosome. As a consequence, the results of one chromosome treatment reflect the accuracy and efficiency of the software algorithm in a relevant way, with a proportional total reduction in time.

In this article, we assessed the difference between alignment of reads generated from a single chromosome against a corresponding reference chromosome, and against an entire reference genome. Then we generated reads from a whole genome, and compared their distribution with the above cases. We also compared the proportion of mismatched reads in each case above, and visualized the mismatches' distributions.

All the cited studies were carried out using statistical metrics based on the count of correctly, incorrectly and unmapped reads. As we discovered, such metrics are not sufficient for a comprehensive assessment of the mapping quality. They do not take into account how well the recovered alignment repeats the initial distribution of the read density. According to our measurements, wrongly mapped full-defined reads range from $0.2 \%$ to $3.8 \%$, and lost reads range from $0 \%$ to $9.5 \%$ of the total number, depending on the software and conditions. In terms of repetition of the distribution density, such a slight fraction can be neglected, but only if these reads are distributed evenly. If 'wrong'reads for some reason are assembled into regions with heightened read density, or lost and matched to other chromosomes reads were initially located in compact regions of the original sequence, it may cause biases and corresponding wrong conclusions. To take into account the influence of this factor, we introduced a new metric, and showed its practical significance.

\section{Methods}

\section{Synthetic data}

For our purposes, we needed a simulator of uniformly distributed DNA reads with the following requirements: (i) all reads should be fully defined, (ii) it should generate the sequence in two formats: in FastQ as an initial data for the verified aligner, and in SAM/BAM/BED as a reliable comparison template ('precise alignment'). Under a fully defined read, we mean a read that (a) carries information in its original actual position, (b) has the maximum quality value, (c) does not include any ambiguous reference characters ('N'). Such set of reads may be called 'ideal'.

There is a wide choice of simulators for genomic next generation sequencing. A brilliant and almost exhaustive review of more than twenty of them was carried out by M.Escalona et al. [10]. We also found two simulation- related sources, not included in the review: MAQ [11] and SEAL [12].

However, none of the considered tools meets our requirements to the fullest. Therefore we chose the ChIPseq simulator isChIP [13], which is able (in the 'control' mode) to generate the sequences that completely meet our needs. The program was used with default settings, the only exception was the refinement of the 'number of cells' option, which was 10 (for single end mode) and 5 (for paired end one).

Our dataset was derived from control test ('input') generated by isChIP on UCSC mouse reference genome (mm9), with the length of reads stated as $50 \mathrm{bp}$.

To approximate real life condition, the first data set is composed of almost 4 million reads randomly and uniformly drawn from the reference chromosome 1 .

The second data set consist of 51 million reads drawn from the whole reference genome.

As we have the 'precise alignment' with known locations of every drawn read by simulation, let us call this sample the 'Gold Standard Alignment' (GSA), 'taking a leaf out of book' [9].

\section{Implementation}

We applied our benchmarking tests on 7 open source DNA sequencing mapping tools, namely Bowtie (1.1.1) [14], Bowtie2 (2.2.4) [15], BWA (0.7.5 and 0.7.12 applying two algorithms) [16], MAQ (0.7.1) [11], MOSAIK (2.2.3) [17], SMALT (0.7.6) [18]. Another 2 aligners have been rejected after repeated trials: BBMap (36.32) and gnumap (3.0.2). We also tried to compile BLASR, GSNAP and STAR, but without success.

The default settings were used for the software mentioned above. The only exception was an increase in memory per thread up to $800 \mathrm{mb}$ in case of Bowtie.

Commonly, methods based on binary classification: 'aligned' vs 'non-aligned' reads, are used to assess mapping accuracy. Indeed as we have a GSA we are able to quantify the aligned reads by computing so called 'confusion matrix' of performance: amount of true positives (TP), reads aligned correctly; false positives (FP), reads aligned wrongly; false negatives (FN), reads not aligned but belonging to the simulated GSA. The true negatives (TN) are legitimately rejected reads, they are a degenerate case in this context. We can then calculate different statistical measures, derived from confusion matrix (such as mapping accuracy and recall), to evaluate the performance of mappers.

However, not only these statistical measures are important in assessing of an aligner's performance, but also an aligned reads' density distribution, which reflects evenness and variance of a coverage. We want to check whether the recovered (obtained after alignment) read density is similar to the original one. 
Therefore, for the most comprehensive evaluation of the mapping accuracy, two complementary measures should be used: the measure of robustness/accuracy is one, and the measure of distribution similarity as another.

\section{Measure of test accuracy}

As a measure of test accuracy we will use the $F_{1}$ value, following the example of Farzana et al. [9].

The $F_{1}$ value can be interpreted as a weighted average of the precision and recall:

$$
\mathrm{F}_{1}=2 \times\left(\frac{p \times r}{p+r}\right)
$$

where $p$ denotes the positive predictive value (precision), and $r$ denotes the true positive rate (sensitivity, or recall). The $F_{1}$ value varies between 0 and 1 , indicating highest accuracy when it is 1 . Precision and recall are computed from the confusion matrix according to: $p=\mathrm{TP} /(\mathrm{TP}+\mathrm{FP}), r=\mathrm{TP} /(\mathrm{TP}+\mathrm{FN})$.

Note that we interpreted TP, FP and FN definitions in a slightly different way than Farzana et al. While the authors defined them in terms of true or wrong location only, we also took into consideration the mismatches per read, i.e. the number of different nucleotides as compared with referenced fragment.

Specifically, we defined true positives as a number of all reads without mismatches, not only reads mapped exactly to the same position as prescribed by GSA. The hits without mismatches, but mapped on wrong positions are the cases of multiple mappings due to paralogous sequences in the reference genome, so-called multi-map reads, or simply multi-reads.

Although most of the tested tools reveal multi-reads and assign them a low-quality value, it is impossible to identify which short sequence between two identical is 'true '. As a result, some of the exactly matched reads also belong to multi-map and are awarded by low value. In our measurements the number of such detected 'wrong-placed'can reach $3.5 \%$ of the total initial number, and about the same percentage of low-quality reads had been included in the 'right-placed'group. So, to achieve the fair evaluation, all multi-reads should be either included or excluded from the TP enumeration. Here we include multi-reads.

Accordingly, we assigned false positive as the number of reads with mismatches and mapped to a different position than the one defined by the global alignment. We will call them simply mismatched reads.

False negative is the number of lost reads, i.e. reads rejected by the aligner due to using heuristics in the mapping algorithm or limitations of the default options.

\section{Measure of distribution similarity}

As a measure of the distributions' similarity we will use a relative standard deviation of read density distribution from sample, CVS.

We defined the 'read density distribution' (or simply 'density profile') as a discrete set of numbers (frequencies) of the base adjacent regions on the reference chromosome (windows), containing 1, 2, 3, etc. reads. To avoid windows with meaningless density, before splitting the sequence into windows, we removed all the gaps (i.e. regions in a reference chromosome completely filled with ambiguous reference characters ' $N$ ') from it. In our experiments, the length of the window was $200 \mathrm{bp}$, the minimum length of the gap was $50 \mathrm{bp}$. Besides, the read was considered belonging to window if it's centre was placed within the window. Such adjustment allows to keep the total amount of reads permanent, so we obtain the actual density.

The density profiles were computed for the original sequence and for the results of each aligner.

To compare these profiles we define an analogue of coefficient of variation, using GSA's counts in a window, assuming it is what we expect from a good aligner's job:

$$
\mathrm{CVS}=\frac{1}{\mu} \sqrt{\frac{1}{\mathrm{~N}} \sum_{\mathrm{i}=1}^{\mathrm{N}}\left(\mathrm{x}_{\mathrm{i}}-\mathrm{X}_{\mathrm{i}}\right)^{2}},
$$

Here CVS denotes the 'coefficient of variation of a sample, $x_{i}$ is the number of windows containing $i$ reads obtained by an aligner, $X_{i}$ denotes the number of GSA windows containing i reads, $\mu$ denotes the GSA's value across all window frequencies, $\mathrm{N}$ is the total number of window frequencies. The minimum value $\mathrm{CVS}=0$ means no deviation from the GSA. The maximum value is not limited.

All the computational work was carried out by our software DenPro [19].

As we mentioned before, first of all we are interested in 'false gaps'and 'false peaks'. The increased frequency of windows with a small number of reads means the abundance of deeps, while the increased frequency of windows with a large number of reads indicates the falsely enriched areas.

'False gaps' and 'false peaks' appear as a deviation of the density profiles after aligning from the initial in the range of window counts with a small number of reads and with large ones, respectively. In our data, zeronumbered windows were missing, although with smaller densities characteristic of 'poor' experimental data and a shorter window length, they may well be present. The observed deviations of the restored density profiles for the windows with a small number of reads, were limited to the frequency of windows containing a single read. We called this singular range 'head'. Deviations for the windows with a large number of reads have become noticeable when frequency was 13 reads per window or 
more. We called this range 'tail'. For a numerical evaluation of these phenomena, it makes sense to calculate the coefficient of variation in these ranges separately, along with the total $\mathrm{CV}$. Respectively, we called these coefficients 'head CVS', CVSh, and 'tail CVS', CVSt:

$$
\begin{aligned}
\text { CVSh } & =\frac{1}{\mu} \sqrt{\left(x_{1}-X_{1}\right)^{2}}, \text { CVSt } \\
& =\frac{1}{\mu} \sqrt{\frac{1}{N-12} \sum_{i=13}^{N}\left(x_{i}-X_{i}\right)^{2}}
\end{aligned}
$$

Here $\mu$ denotes the GSA's value across the specified ranges.

Three series of tests have been conducted: (i) mapping of reads from a single chromosome to a reference chromosome, (ii) mapping of reads from a single chromosome to a reference genome, and (iii) mapping of reads from a whole genome to a reference genome. For convenience, let us call these series case1, case 2 and case3.

Case1 is refined and unpractical, but it encourages software to produce the highest possible, 'ideal'result, and could be very appealing for the benchmarking of aligners and peak callers.

Case 2 is unpractical too, it is interesting as a light candidate for the benchmarking of peak callers.

Case 3 corresponds to real life conditions.

\section{Results}

Test distribution similarity with GSA.

\section{Case 1: Mapping of reads from single chromosome to} reference chromosome

Bowtie and Bowtie2 have been excluded from this case since they are supplemented by pre-build reference genome indexes. We also evaluated BWA 0.7.5 in this series only, mainly to demonstrate the difference between the old and latest versions of program. Hereinafter we used the latest version BWA 0.7.12 as more accurate. Wherever we mention BWA without version indication we refer to BWA 0.7.12.

CVSs for case1 are shown in Fig. 1A. We can observe the very different level of CVS for different aligners. Only MAQ, and partly BWA-mem showed the perfect coincidence with the original read density distribution in both single end (SE) and paired end (PE) modes. In SE mode SMALT and partly BWA-samse also showed the good results, while BWA 0.7.5 and MOSAIK demonstrated a high level of unexpected depleted and enriched regions, accordingly. In PE mode however, the picture is changed: MOSAIK showed a heightened level of 'false peaks'and 'false gaps', and SMALT - a heightened level of 'false peaks', unlike other tools.

As an illustration of these issues, we give direct combined images of SE and PE density profiles (Fig. 2). Falsely enriched regions of the two aligners become obvious in the fragment of coverage graph (Fig. 3). In case of BWA 0.7.5 these 'false peaks'are concentrated at the beginning of the chromosome, while MOSAIK produces such enrichments along the entire chromosome.

But these results should be considered as insufficient without taking into account the alignment quality score.

We found that more than $90 \%$ of exactly matched SE reads had the highest possible score for all examined aligners (except MOSAIK with its value of $66 \%)$. For PE reads the same minimum value was 94\% (15\% in case of MOSAIK). Only Bowtie marks all its hits by the maximum score value. Bowtie2 and MAQ assign the lowest possible quality score to all, BWA - to almost all of mismatched and multihits. MOSAIK and SMALT mark mismatched and multi- reads with low scores.

All the aligners, except Bowtie2, assign zero score for the most doubtful located reads. It is interesting

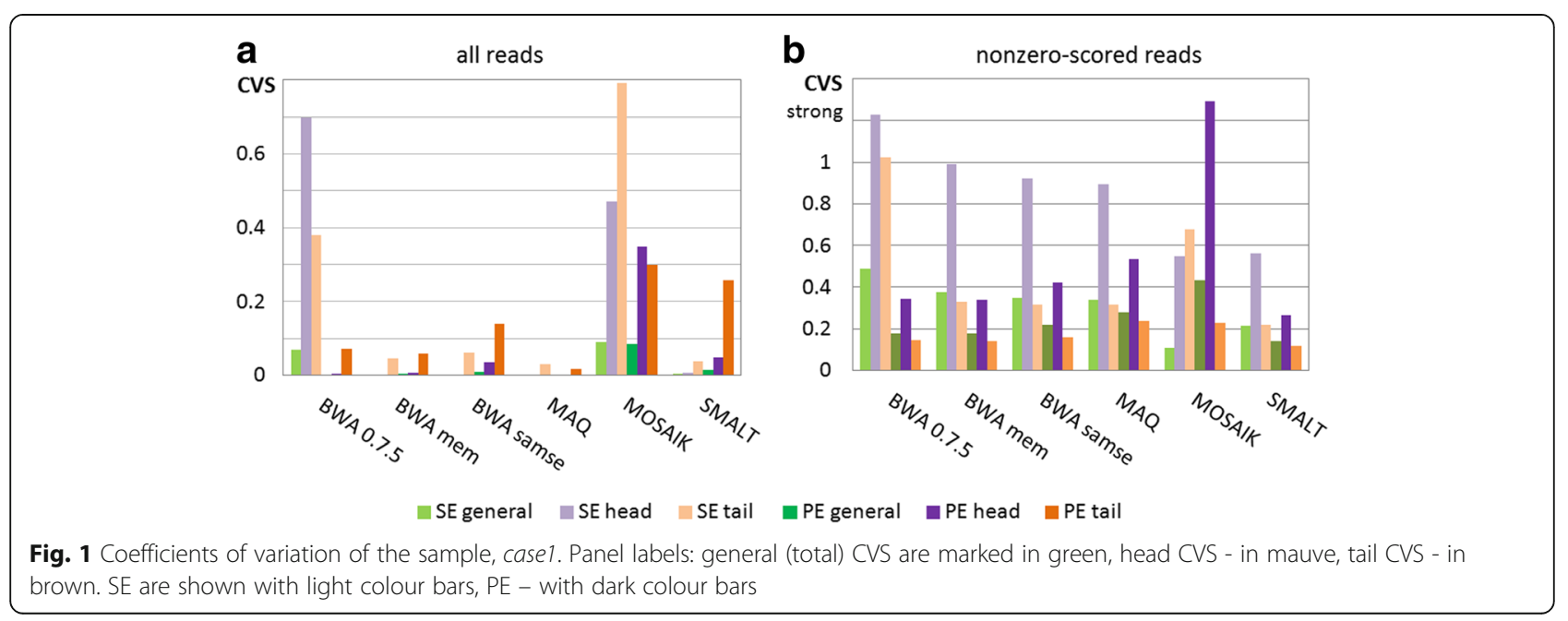



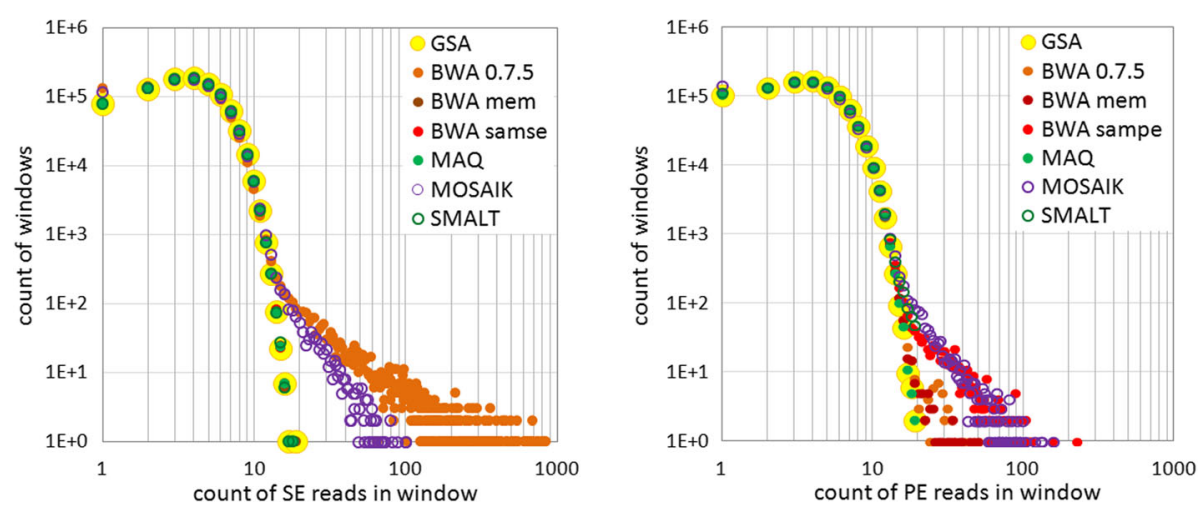

Fig. 2 Density profiles for mapping of reads in log-log coordinates, casel

to know what effect such zero-scored hits have on the density profile. We called 'reliable' sequences with filtered zero-scored reads, and represented them in Fig. 1B.

\section{Case2: Mapping of reads from single chromosome to reference genome}

There is a dramatic change in coverage evenness when aligners map the same data (simulated from a single chromosome) to the whole genome (see Fig. 4A). We can observe an almost equal pattern of synchronous read-depleted regions (gaps) for all aligners. The gaps have characteristic length of 1-10 kpb (see Fig. 5). And the difference between ordinary and 'reliable' distributions became much less (Fig. 4B).

According to CVS measures, in this case all aligned sequences are distinguished by a remarkable similarity between themselves, in contrast to other cases. This is also confirmed by the Pearson correlation: the crosscorrelation coefficients are about 0.98 , while in the other cases they vary from 0.89 to 0.93 (with the exception of MOSAIK, which shows the lower value for all cases). Pearson coefficients were calculated by our software
bioCC [20] and are presented in supplementary tables, along with the remaining results.

\section{Case3: Mapping of reads from genome to reference genome.}

As we mentioned above, this is the most realistic condition, and consequently the most complicated case.MAQ and MOSAIK have been excluded from this series due to the requirement of inappropriate execution time (more than $50 \mathrm{CPU}$ hours on a highperformance computer). Besides MAQ demands additional efforts to split the job because of limited input sequence size (2-3 million reads).

As shown in Fig. 6A, only Bowtie demonstrates a very good coincidence with GSA in both SE and PE modes. BWA and SMALT showed an unexpected high level of false PE 'peaks'. This time, SMALT showed a relative clustering of 'false peaks' at the first third of the chromosome, while BWA generates such peaks evenly along the chromosome. A low 'reliable' CVS levels for Bowtie should not be misleading, since this aligner does not differentiate the quality score (Fig. 6B).

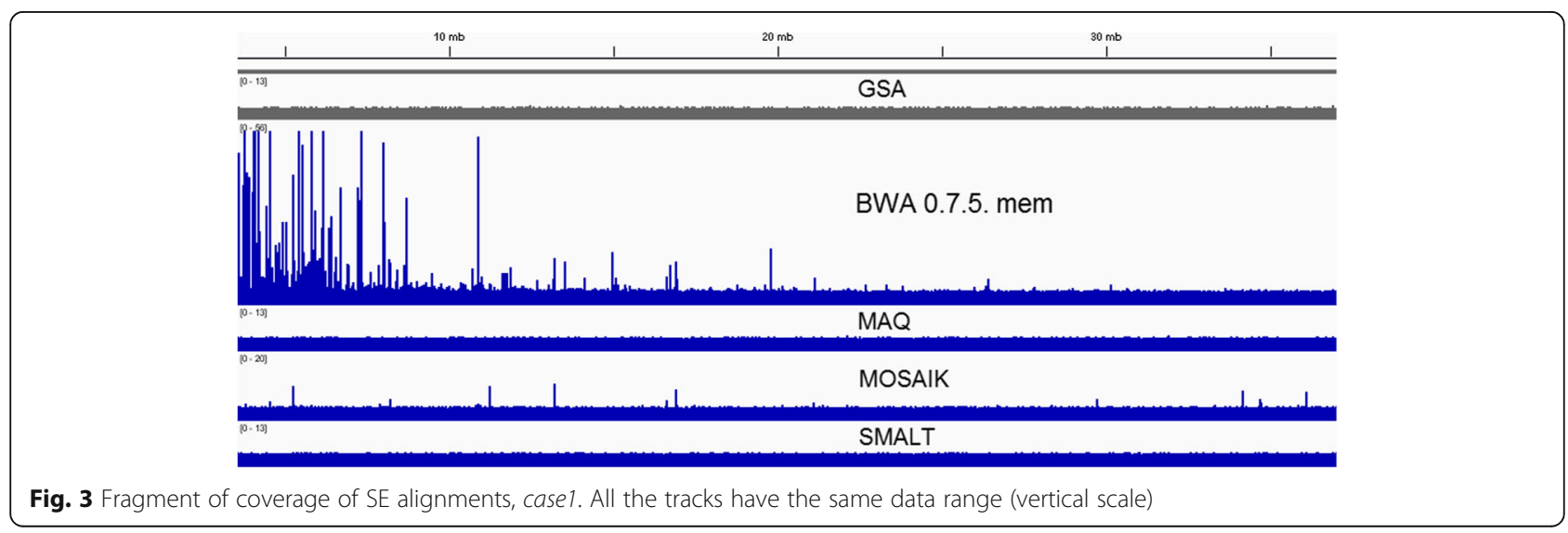




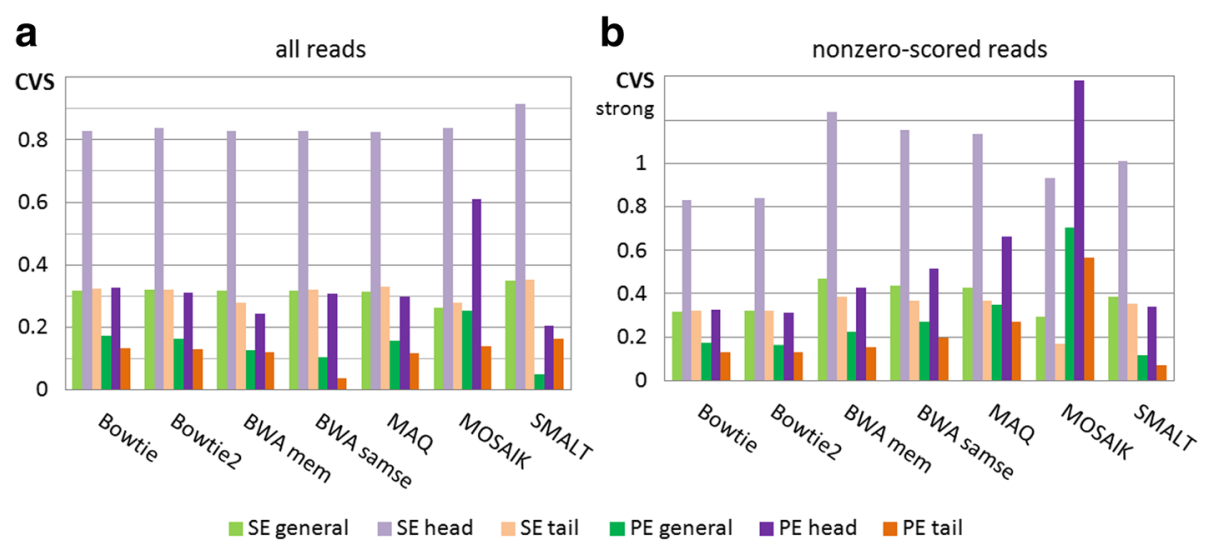

Fig. 4 Coefficients of variation of the sample, case2. Panel labels: general (total) CVS are marked in green, head CVS - in mauve, tail CVS - in brown. SE are shown with light colour bars, PE - with dark colour bars

Note that all calculations were performed for the first chromosome. However, to avoid possible dependency on the chromosome particular structure, we conducted control measurements for two more chromosomes (second and third) in a limited conditions (case1 and case2 in a single end mode), and obtained very consistent results.

\section{Test accuracy}

$F_{1}$ scores for all cases are represented on Fig. 7.

Statistical results for all aligners look fairly even with respect to $F_{1}$ values, and remarkably similar, despite 'false'peaks and gaps. This confirms our statement that the evaluation of aligners based only on confusion matrix (binary statistics) is incomplete.

The low value of $F_{1}$ in the case of MOSAIK PE reads, especially 'reliable', is explained by a relatively high level of lost reads. Besides, almost all its mismatched reads have non-zero score. Actually, it is a specific shortcoming of PE aligning for this tool. BWA 0.7.5 has the highest level of lost $\mathrm{SE}$ reads, so it's $\mathrm{F}_{1}$ for $\mathrm{SE}$ reads is the least.

\section{Mismatches statistics}

Since all reads in an input sequence are full-defined, we can extract information about mismatches and their distribution. Please note that all mismatches arrive not because of non-perfect sequencing, but because of non-perfect aligning. Therefore, it is another estimation of the confidence of the mapping. To carry out this work, we developed our own auxiliary program vAlign [21].

\section{Percentage of reads with mismatches}

We counted reads within chromosome 1 with detected mismatches for each aligner. The percentage was calculated with respect to the total number of reads found on the chromosome 1 accordingly. For more information, zero-scored and non-zero-scored reads have been calculated separately. The results are represented in Fig. 8.

The first observation is a high percentage of mismatched reads in case1 for all programs. In this case we can observe the most difference between SE and PE modes, except MOSAIK's outcome.

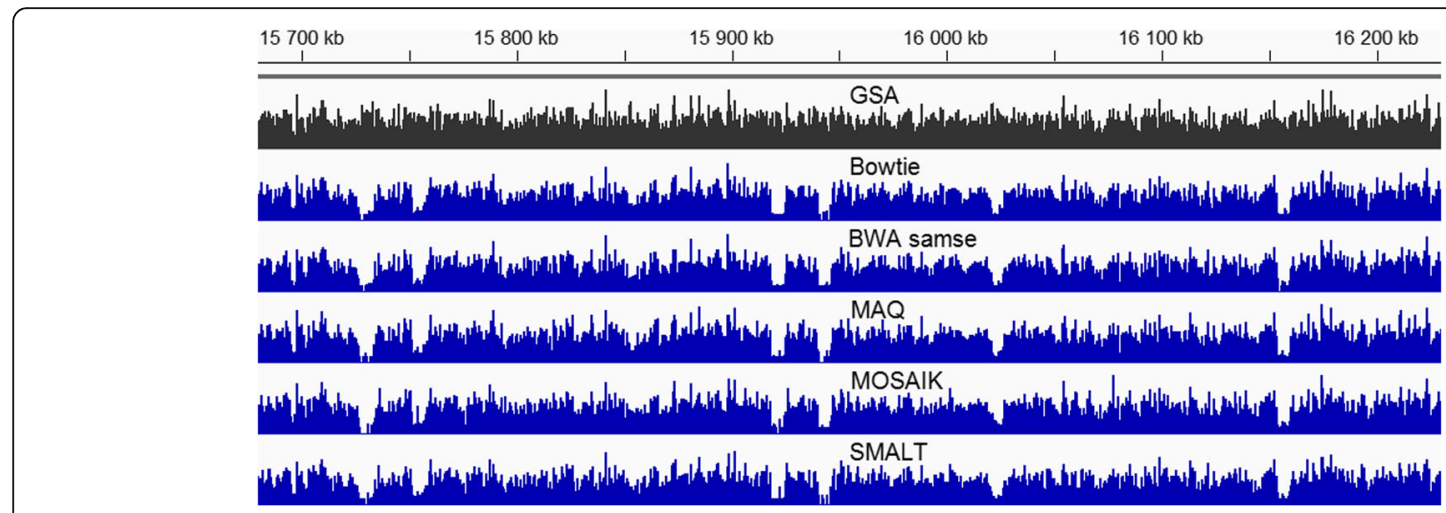

Fig. 5 Fragment of coverage of PE alignments, case2 

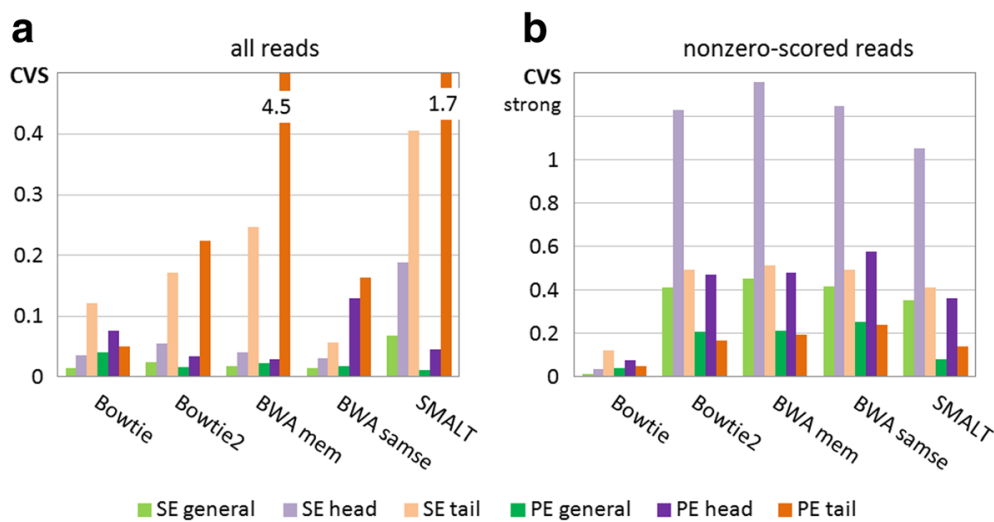

Fig. 6 Coefficients of variation of the sample, case3. Panel labels: general (total) CVS are marked in green, head CVS - in mauve, tail CVS - in brown. SE are shown with light colour bars, PE - with dark colour bars

\section{Specifics of distribution of mismatches along the reads} Among the studies devoted to aligners, we did not find information on the frequency distribution of the mismatches along the read. This issue has no practical significance for users, but may be of interest to aligner developers.

We summarized the mismatches in each nucleotide position for all the readers. The results for case 2 are shown in Fig. 9. Other cases look very similar.

Note that the odd positions in single-end reads did not contain mismatches for all programs, except MOSAIK and SMALT with very little count in odds.
The second remarkable feature is the observed increase in the number of mismatches in the second half of the reads, resembling a close to normal distribution. The maximum of the substitutions is at 38th position.

Also the large value is observed in the two first positions for most of aligners. This effect has a more localized appearance in PE mode.

The third interesting point is that these distributions generally are similar for all tools. On the whole, aligners utilize hashing algorithms or the Burrows-Wheeler transform to search exact matches. Nevertheless each tool uses its own optimization, which, as we see, leads to
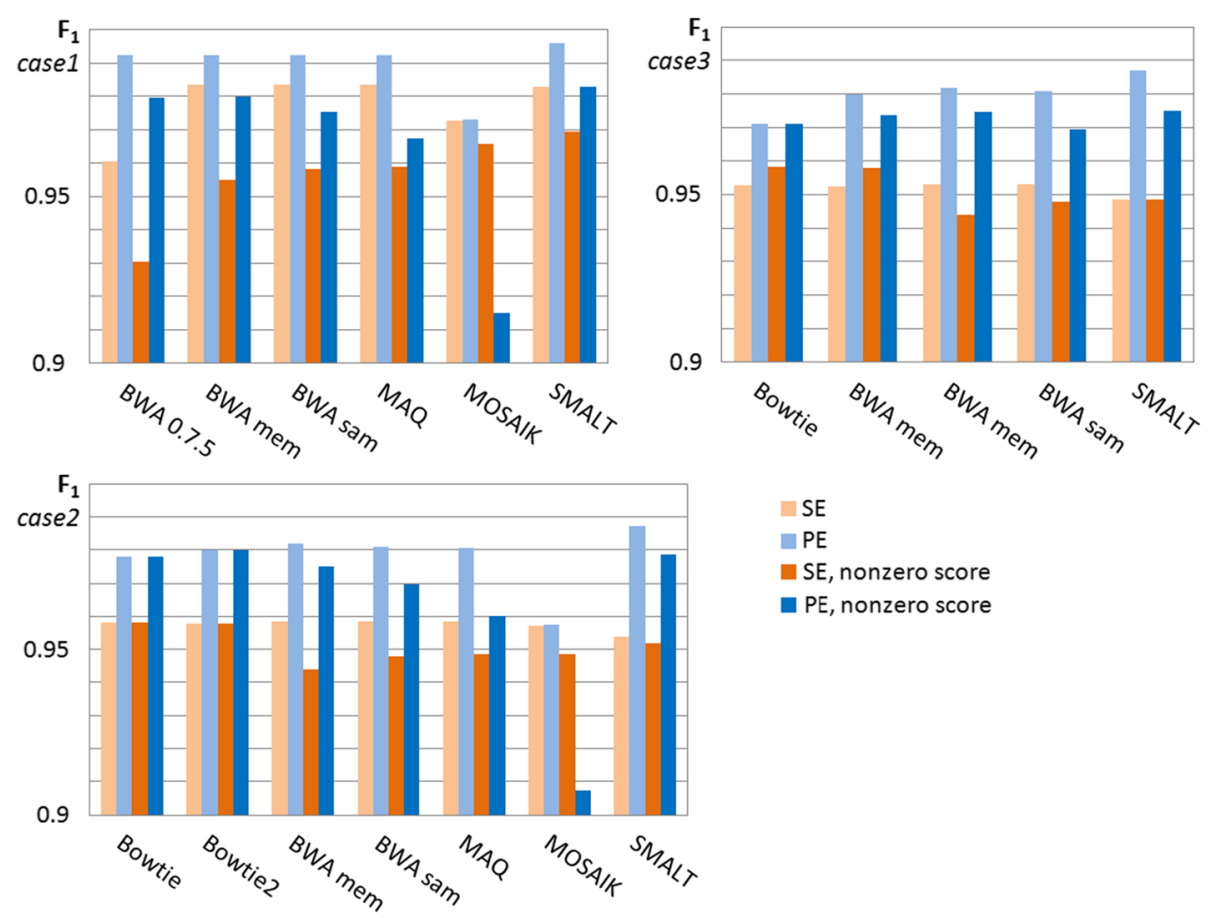

$\square S$
$\square \mathrm{PE}$
$\square \mathrm{SE}$, nonzero score
$\square \mathrm{PE}$, nonzero score

Fig. 7 F1 scores. All reads - light colour bars, non-sero reads ('reliable') - dark colour bars 

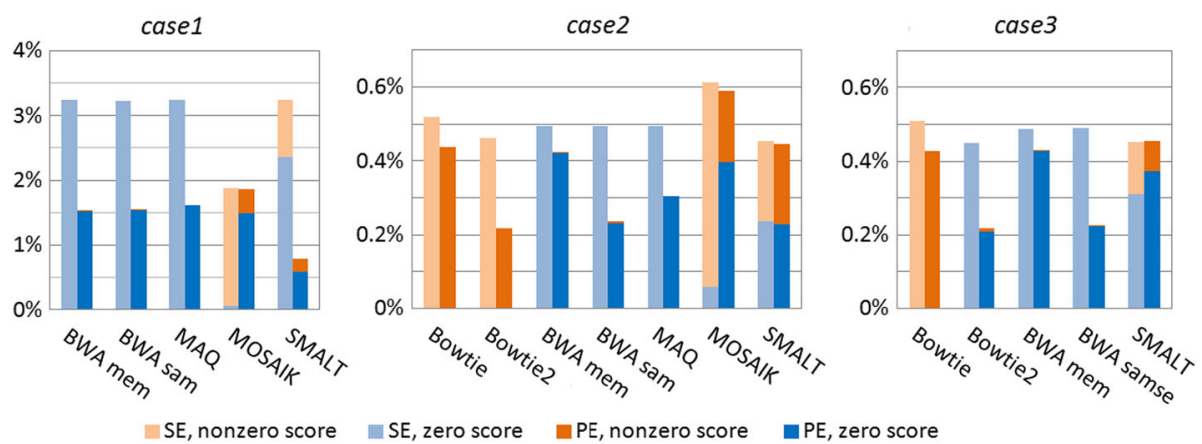

Fig. 8 Percentage of non-zero-scored and zero-scored reads with detected mismatches. SE - pale histogram bars, PE - bright histogram bars

different results. However, this does not concern the distribution of mismatches and the appearance of gaps in case 2 .

\section{Summary of the results}

Using an 'ideal'input sequence, we were expecting that aligning of reads from a single chromosome against a reference chromosome should be the simplest and, as a consequence, the best case for the aligners.

Indeed, 4 out of 6 tools showed $F_{1}$ values exceeding 0.98 for SE reads, and 5 tools showed $F_{1}$ values more than 0.99 for PE reads - these are very good statistical factors. Of these, BWA mem, BWA samse and SMALT showed very good coincidence with GSA in SE mode, while MAQ is perfect in both modes. Other programs generated a sequence that in some variants is distributed in a clearly different way from the sample. And this case really gives a gain in time of mapping from 20 to almost 50 times, as well as more than 10 times in following peak calling.

Mapping a single chromosome to a reference genome leads to system defects (gaps) in alignment, although 6 out of 7 tools showed a good and similar $F_{1}$ values: about 0.96 for SE reads and 0.98 for PE reads.
When aligning a genome against a reference genome, all verified programs produced results statistically similar to the previous case. As for the distribution, BWA samse showed the best coincidence with GSA, in contrast to BWA sampe. Bowtie can be recognized as very good in both modes, despite the slightly lower value of $F_{1}$. Bowtie2 generated a little more of an uneven distribution. And both of BWA mempe and SMALT produced too many false enriched regions in PE mode.

\section{Discussion}

\section{Distribution artefacts}

As we mentioned before, all the sequences in the case 2 are very similar to each other. Consequently, features in the mapping algorithms that led to the obtained difference in cases 1 and 3 , did not appear in case 2 .

The difference in algorithms manifests itself in the way they process multi-reads, and therefore, first of all should be visible within the regions with low mappability [22]. Juxtaposition of the selected sequences with the genome mappability tracks confirmed this assumption, see Fig. 10.

First of all, MAQ demonstrates complete insensitivity to the low mappability regions in case1, and the synchronous coincidence of its 'false gaps' with such regions
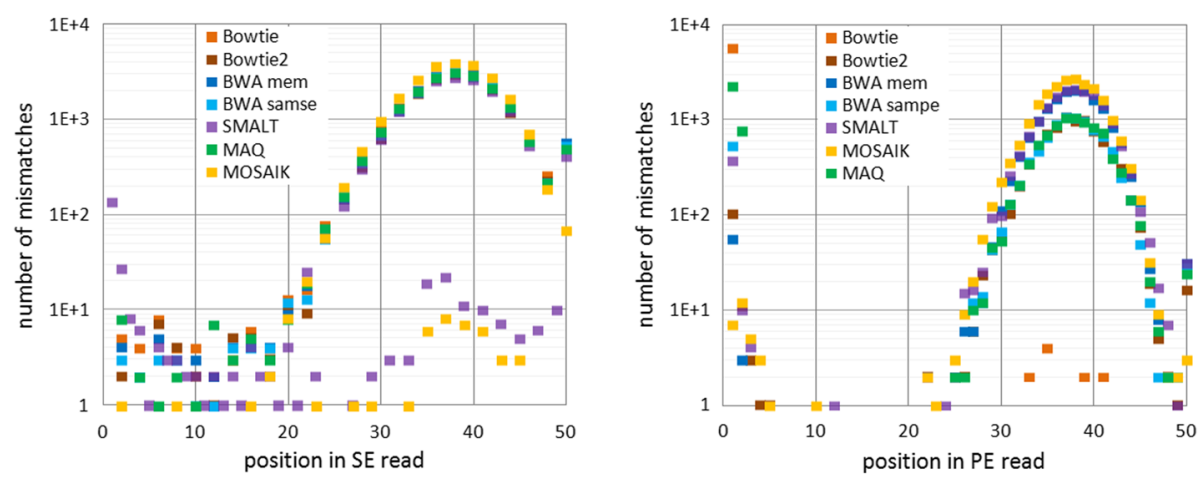

Fig. 9 The frequency of mismatches depending on the position in the read, in log coordinate, case2 


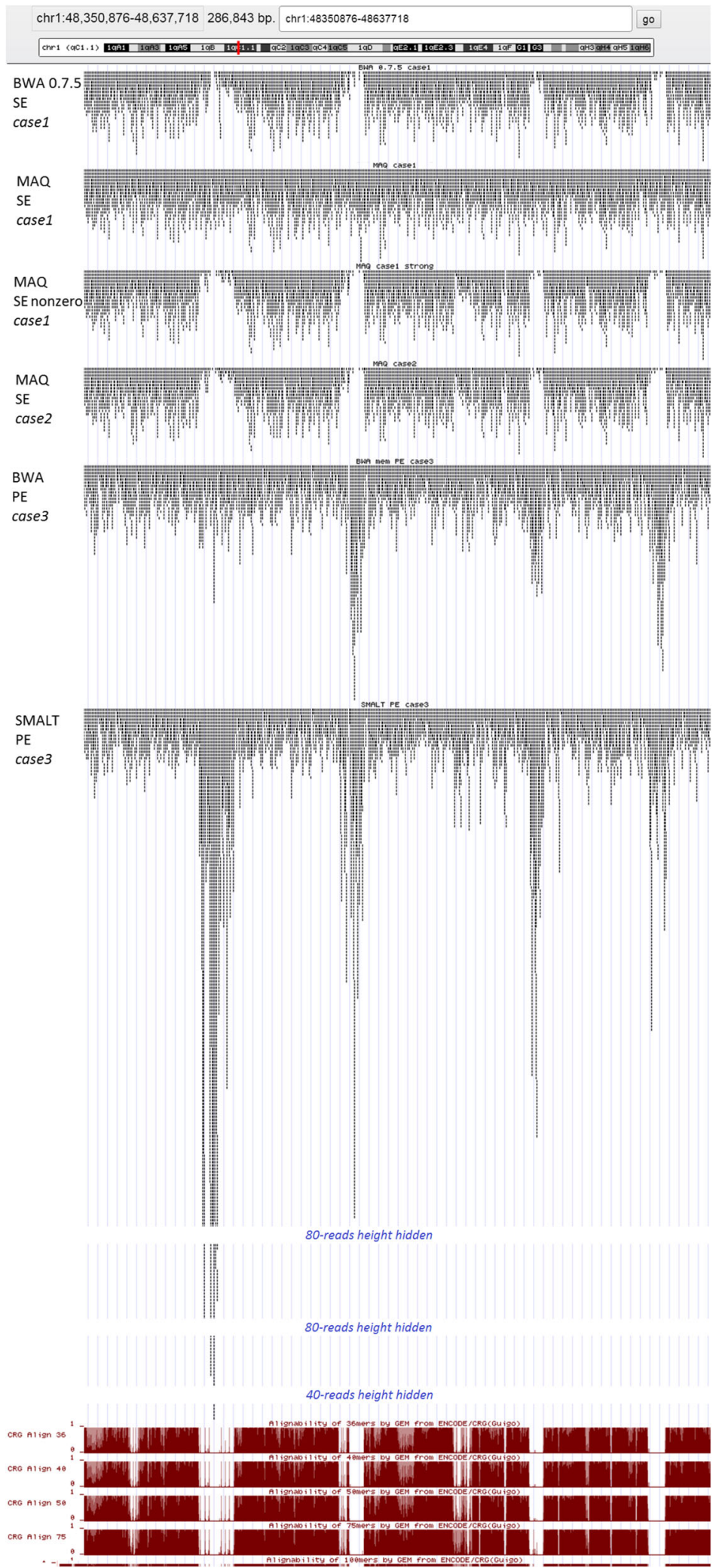

Fig. 10 Fragment of alignments in comparison with low mappability tracks, UCSC genome browser 
in case 2. It is obvious that by referencing a single chromosome to the whole genome, the program first of all tries to best localize the next treated read throughout the whole reference, and matches not uniquely mapped on the first chromosome read to the different chromosomes, as well as other programs. As a result, only the unique hits remain on the first chromosome, which explains the high likeness of all alignments. This conclusion is also confirmed by a perfect correlation between MAQ case 2 and 'reliable' case1, with Pearson coefficient of 0.98 .

Second, we can see that BWA 0.7.5 almost replicates the low mappability regions by its 'false gaps' even in case1. At the beginning of the chromosome, the pattern is reversed: such regions correspond to its intensive 'false peaks', rapidly decreasing in amplitude with distance from the beginning. Obviously, the tool implements the relatively simple algorithm for mapping multi-reads, suggesting their location at the first suitable place, without analyzing the rest of sequence. Other aligners demonstrate more sophisticated approaches, with varying degrees of effectiveness.

A similar strategy is realized by BWA and SMALT in the PE mode in case3, but this time their 'false peaks' are distributed over the entire chromosome. At the same time these tools show a much better result for single end reads, especially BWA samse - actually it is a best SE aligner among the benchmarked in this practical case.

Thus, case 2 can be considered as a non-precise alternative to obtaining low mappability regions, as well as simply removing reads with the minimum admissible score.

\section{The artefacts in the distribution of mismatches}

It possibly should be assumed that the observed effect is related to the seed-and-extend paradigm, followed by Bowtie, BWA and MAQ. In general all these software identify short matches as seeds, and then use the difference extending strategy. In this case, the default length of seed (28 bp for Bowtie and MAQ, and 32 bp for BWA) explains the small number of substitutions in the first half of the read. SMALT implements a different approach, but it also matches a read's segment first. Unfortunately we did not find mention of the seeding in the MOSAIK descriptions. Such assumption is also not enough to explain the normal-shaped form of the distribution of mismatches in the second half of the read, as well as an increased level of substitution rate in the first two positions. This issue requires a deeper study of the aligning algorithms, which is beyond the scope of this article.

The question of the absence of mismatches on odd positions for SE reads also remains open.

\section{Conclusions}

Our study showed that standard binary accuracy statistical measures are insufficient to assess the mapping performance. While having a comparable standard binary statistical metrics, all tested programs demonstrated significant differences in density distribution profiles. These differences are directly related to some shortcomings of the implemented matching algorithms, therefore, our modified $\mathrm{CV}$ can be considered as essential metric even in general-purpose alignments evaluation.

Note that gene regulatory regions in general have lower text complexity and corresponding lower mappability than protein-coding regions [23]. Biases in genomic nucleotide context and low complexity regions add noise to the mapping [24].

Observable deviations of the density of alignment from the sample have different significance. While 'false' peaks are completely eliminated by peak callers, the 'false' gaps can hide the real regions of interest. But 'false peaks' are formed due to 'false gaps', and vice versa.

Therefore, the best recommendation is to choose an aligner with the minimum value of both 'head' and 'tail' CVS. From this point of view and within the limits of the accepted conditions, in particular, while mapping a single chromosome, such tools are MAQ, BWA mem (in both modes) and BWA samse (for SE reads) work very good. When mapping a genome - BWA samse (for SE reads) and Bowtie (in both modes) are recommended.

We also showed some peculiarities of the distribution of mismatches along the reads. The pattern of error growth to the end of reads was commonly attributed to the accumulation of errors during sequencing. However, in our case there are no sequencing errors. This pattern seems to be common for all aligners, regardless of the various algorithms they are based on.

\footnotetext{
Acknowledgements

We would like to thank the anonymous reviewer for his/her suggestion on low mappability regions. Authors are grateful to Dr. Yuriy E. Herbeck and Anton $\mathrm{V}$. Tsukanov for help with the manuscript processing. The computational work was performed on SSCC ICMMG SB RAS, Novosibirsk.
}

\section{Funding \\ The computer code development and biodiversity research were supported by the Ministry of Education and Science of the Russian Federation grant \#14.W03.31.0015. The algorithm development was supported by ICG SB RAS budget project 0324-2018-0017. The software testing on supercomputer cluster was supported by the Russian Science Foundation (grant no. 16-14 10216). The publication costs for this article were funded by RSF (grant no. 16-14-10216).}

\section{Availability of data and materials}

All initial sequences and tables with the results of calculations and graphs are available at https://figshare.com/s/d75a2199eda36a4ce2ae.

\section{About this supplement}

This article has been published as part of BMC Genomics Volume 19 Supplement 3, 2018: Selected articles from Belyaev Conference 2017: genomics. The full contents of the supplement are available online at https://bmcgenomics.biomedcentral.com/articles/supplements/volume-19supplement-3. 


\section{Authors' contributions}

FN conceived and constructed the method, developed software, conducted numerical experiments and wrote the paper. IA reviewed the statistical methods and the paper. NB reviewed the paper. MG supported computer cluster testing. YO organized computational experiments and specified the paper. All authors approved the final manuscript.

\section{Authors' information}

FN is an independent software developer, employed by Novosibirsk State University. IA is a Daphne Jackson Fellow sponsored by the Babraham Institute and BBSRC. NB is researcher at University of Hertfordshire. MG is a Postdoc at ICG SB RAS. YO is a senior scientist at ICG SB RAS, IMBR RAS, and professor at Novosibirsk State University.

\section{Ethics approval and consent to participate}

Not applicable.

\section{Consent for publication}

Not applicable.

\section{Competing interests}

The authors declare that they have no competing interests.

\section{Publisher's Note}

Springer Nature remains neutral with regard to jurisdictional claims in published maps and institutional affiliations.

\section{Author details}

${ }^{1}$ Novosibirsk State University, Pirogova, 1, Novosibirsk 630090, Russia. ${ }^{2}$ Wellcome Trust Sanger Institute, Cambridge, UK. ${ }^{3}$ Babraham Institute, Cambridge, UK. ${ }^{4}$ University of Hertfordshire, Hertfordshire, UK. Institute of Cytology and Genetics SB RAS, Novosibirsk, Russia. ${ }^{6}$ Institute of Marine Biology Researches of RAS, Sevastopol, Russia.

\section{Published: 9 February 2018}

\section{References}

1. Soon WW, Hariharan M, Snyder MP. High-throughput sequencing for biology and medicine. Mol Syst Biol. 2013;9:640. https://doi.org/10.1038/ msb.2012.6.

2. Ruffalo M, LaFramboise T, Koyutürk M. Comparative analysis of algorithms for next-generation sequencing read alignment. Bioinformatics. 2011;27(20): 2790-6. https://doi.org/10.1093/bioinformatics/btr477.

3. Fonseca NA, Rung J, Brazma A, Marioni JC. Tools for mapping highthroughput sequencing data. Bioinformatics. 2012;28(24):3169-77.

4. Schbath S, Martin V, Zytnicki M, Fayolle J, Loux V, Gibrat JF. Mapping reads on a genomic sequence: an algorithmic overview and a practical comparative analysis. J Comput Biol. 2012;19(6):796-813. https://doi.org/10. 1089/cmb.2012.0022

5. Hatem A, Bozdağ D, Toland $A E$, Çatalyürek ÜV. Benchmarking short sequence mapping tools. BMC Bioinformatics. 2013;14:184. https://doi.org/ 10.1186/1471-2105-14-184

6. Shang J, Zhu F, Vongsangnak W, Tang Y, Zhang W, Shen B. Evaluation and comparison of multiple aligners for next-generation sequencing data analysis. Biomed Res Int. 2014;2014:309650. https://doi.org/10.1155/2014/309650.

7. Otto C, Stadler PF, Hoffmann S. Lacking alignments? The next generation sequencing mapper segemehl revisited. Bioinformatics. 2014;30(13):1837-43. https://doi.org/10.1093/bioinformatics/btu146.

8. Caboche S, Audebert C, Lemoine Y, Hot D. Comparison of mapping algorithms used in high-throughput sequencing: application to ion torrent data. BMC Genomics. 2014;15:264. https://doi.org/10.1186/1471-2164-15-264.

9. Rahman F, Hassan M, Kryshchenko AM, Dubchak I, Alexandrov N, Tatarinova T. BenchNGS: An approach to benchmark short reads alignment tools. bioRxiv. 2015; https://doi.org/10.1101/018234.

10. Escalona M, Rocha S, Posada D. A comparison of tools for the simulation of genomic next-generation sequencing data. Nature Review Genetics. 2016; 17:459-69. https://doi.org/10.1038/nrg.2016.57.

11. Li H, Ruan J, Durbin R. Mapping short DNA sequencing reads and calling variants using mapping quality scores. Genome Res. 2008;18(11):1851-8. https://doi.org/10.1101/gr.078212.108.
12. Ruffalo M, LaFramboise T, Koyutürk M. Comparative Analysis of algorithms for next-generation sequencing read alignment. Bioinformatics. 2010;27(20): 2790-6. https://doi.org/10.1093/bioinformatics/btr477.

13. In silico ChIP-seq simulator https://github.com/fnaumenko/isChIP

14. Langmead B, Trapnell C, Pop M, Salzberg SL. Ultrafast and memory-efficient alignment of short DNA sequences to the human genome. Genome Biol. 2009;10(3):25. https://doi.org/10.1186/gb-2009-10-3-r25

15. Langmead B, Salzberg SL. Fast gapped-read alignment with bowtie 2. Nat Methods. 2012;9(4):357-9. https://doi.org/10.1038/nmeth.1923.

16. Li H, Durbin R. Fast and accurate short read alignment with burrowswheeler transform. Bioinformatics. 2009;25(14):1754-60. https://doi.org/10. 1093/bioinformatics/btp324.

17. Lee WP, Stromberg MP, Ward A, Stewart C, Garrison EP, Marth GT. MOSAIK: a hash-based algorithm for accurate next-generation sequencing short-read mapping. PLoS One. 2014;9(3):e90581. https://doi. org/10.1371/journal.pone.0090581.

18. Ponstingl $H$, Ning Z . SMALT - a new mapper for DNA sequencing reads. f1000research. 2010; https://f1000research.com/posters/327

19. Naumenko F. Read Density Profile calculator. https://github.com/ fnaumenko/DenPro

20. Naumenko F. Correlation calculator for bioinformatics data. https://github. com/fnaumenko/bioCC

21. Naumenko F. Mismatches distribution calculator. https://github.com/ fnaumenko/vAlign

22. Derrien T, Estellé J, Marco Sola S, Knowles DG, Raineri E, Guigó R, Ribeca P. Fast computation and applications of genome Mappability. PLoS One. 2012; 7(1):e30377. https://doi.org/10.1371/journal.pone.0030377.

23. Orlov YL, te Boekhorst R, Abnizova II. Statistical measures of the structure of genomic sequences: entropy, complexity, and position information. J Bioinforma Comput Biol. 2006;4:523-36.

24. Goh WS, Orlov Y, Li J, Clarke ND. Blurring of high-resolution data shows that the effect of intrinsic nucleosome occupancy on transcription factor binding is mostly regional, not local. PLoS Comput Biol. 2010;6(1):e1000649. https://doi.org/10.1371/journal.pcbi.1000649.

\section{Submit your next manuscript to BioMed Central and we will help you at every step:}

- We accept pre-submission inquiries

- Our selector tool helps you to find the most relevant journal

- We provide round the clock customer support

- Convenient online submission

- Thorough peer review

- Inclusion in PubMed and all major indexing services

- Maximum visibility for your research

Submit your manuscript at www.biomedcentral.com/submit
Biomed Central 